\title{
25 Research Square \\ Clinical characteristics and prognostic factors of 148 COVID-19 cases in a secondary epidemic area
}

Hongying Shi

Wenzhou Medical University

Lingyun $\mathrm{He}$

Wenzhou Medical University Second Affiliated Hospital

Weijian Sun

Wenzhou Medical University Second Affiliated Hospital

Jingxuan Xu

Wenzhou Medical University Second Affiliated Hospital

\section{Molin Wang}

Harvard University T H Chan School of Public Health

Xiaodong Chen

Wenzhou Medical University Second Affiliated Hospital

Hongmei Zeng

Chinese Academy of Medical Sciences and Peking Union Medical College

\section{Weiteng Zhang}

Wenzhou Medical University Second Affiliated Hospital

\section{Xiangwei Sun}

Wenzhou Medical University Second Affiliated Hospital

Yuanbo Hu

Wenzhou Medical University Second Affiliated Hospital

Weiping Ji

Wenzhou Medical University Second Affiliated Hospital

Xiangyang Xue

Wenzhou Medical University

Xian Shen ( $\nabla$ shenxian5166@gmail.com )

Wenzhou Medical University Second Affiliated Hospital https://orcid.org/0000-0003-1164-4832

\section{Research article}

Keywords: clinical epidemiology, COVID-19, prognosis, SARS-CoV-2, outbreak

Posted Date: May 22nd, 2020 
DOI: https://doi.org/10.21203/rs.3.rs-28362/v1

License: (a) (i) This work is licensed under a Creative Commons Attribution 4.0 International License. Read Full License 


\section{Abstract}

Background: COVID-19 has rapidly spread worldwide; however, the prognostic factors of COVID-19 have not been well described. Based on the prospective medical records in a secondary epidemic area, we examined the epidemiological, clinical characteristics, and explored the prognostic factors of COVID-19 patients.

Methods: This retrospective, multi-center cohort study included consecutive cases with laboratoryconfirmed COVID-19 from January 22 to February 22, 2020 in Wenzhou, China. All cases were followed up to discharge, death, or up to March 16, 2020. Demographic, clinical, laboratory, treatment, and prognosis data were extracted with standardized form, and compared between severe and non-severe cases. Logistic regression and Cox regression were used to explore the factors associated with ICU admission and hospital stay, respectively.

Results: The epidemic curve showed that the duration of the whole epidemic in this secondary epidemic area was relatively short. Of the 148 included cases, 29 were severe cases. The median age was 52 years (range, 10-93 years), $62.2 \%$ were male, and $60.8 \%$ were overweight or obese. Fever (81.8\%) and cough (61.5\%) were the most common symptoms. Importantly, $25.0 \%$ had no clear contact history. The median length of hospital stay was 20 days (95\% Cl: 19-21), and $5.4 \%$ of patients were admitted to ICU, and one patient died. Compared with the non-severe cases, the severe patients were older, had a longer hospital stay, and had higher proportions of smoking, laboratory abnormalities, and ICU risk. Elevated alanine aminotransferase was independently associated with ICU admission; however, type of antiviral drugs had no significant association with duration of hospital stay. Older age, faster respiratory rate, smoking, and comorbidity tended to have higher risk of ICU admission and longer duration of hospital stay.

Conclusions: The second-generation cases had atypical symptoms, and one quarter of cases had no clear contact history with cases, indicating the importance of strict preventive and control measures. Abnormal liver function, faster respiratory rate and smoking may be associated with worse prognosis of COVID-19; however, there was no convincing evidence for an association between antiviral therapy and length of hospital stay.

\section{Introduction}

The ongoing outbreak of pneumonia associated with a novel coronavirus began with the first reported case on 12 December 2019[1] in Wuhan, Hubei province. On February 12, 2020, the World Health Organization (WHO) officially named the disease as coronavirus disease 2019 (COVID-19). It was then found in different regions of China and at least 143 other countries[2]. Mounting epidemiological evidence suggests COVID-19 is more contagious than either SARS-CoV or MERS-CoV. The WHO declared that the COVID-19 pneumonia epidemic constituted a pandemic on March 11[3]. As of April 3, there were 972,303 confirmed cases and 50,322 deaths worldwide [4]. Several studies [5-7] documenting the clinical features of patients with laboratory-confirmed COVID-19 have been published, and most of them were 
from Wuhan, with a relatively high proportion of severe cases and complications. There are few clinical studies of COVID-19 cases in secondary epidemic areas, where clinical manifestations might be different with those of the Wuhan cases.

With the rapid global spread of the virus, exploring the clinical characteristics of patients outside Wuhan is necessary to advance our understanding of COVID-19 in secondary epidemic areas and provide information that could aid in COVID-19 prevention and treatment worldwide. Many people from Wenzhou, a city on the southeast coast of Zhejiang province, work in Wuhan. Consequently, this city suffered greatly in the COVID-19 outbreak, with the largest number of confirmed cases outside Hubei province in China. Therefore, we aimed to examine the epidemiological, clinical characteristics of confirmed COVID19 patients, and we also explored the prognostic factors of COVID-19 patients in this city in China.

\section{Methods}

\subsection{Participants}

This study included all laboratory-confirmed COVID-19 cases in two of the top three hospitals in Wenzhou (the First and Second affiliated Hospitals of Wenzhou Medical University), from January 22 to February 22,2020 . They were categorized based on the Chinese diagnosis and treatment guidelines of new coronavirus infection (seventh edition)[8] as mild, moderate, severe, and critically severe.

All cases were followed up to discharge, or the end of this study (March 16, 2020). Discharge criteria were: negative results from two consecutive RT-PCR tests, negative routine stool tests, and no obvious positive signs on imaging examination.

\subsection{Data collection}

Using a standardized data collection form (modified from the case record form of novel coronavirus shared by the WHO), we extracted the following information: (1) Demographic characteristics, including age, sex, height, and weight. Based on the Chinese standard for obesity in adults, patients were classified

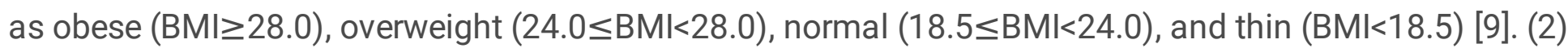
History of Wuhan exposure: patients were classified into four groups: direct Wuhan exposure (patient had recently resided in or visited Wuhan); indirect Wuhan exposure (close contact with someone who had been to Wuhan during this outbreak); exposure to other confirmed patients; and no clear exposure history. (3) Clinical symptoms, and the durations from disease onset to hospital admission. (4) Laboratory findings on admission, such as complete blood count, blood chemistry, liver and renal function, coagulation test, and levels of C-reactive protein, procalcitonin, and cardiac enzymes (lactate dehydrogenase and creatine kinase). To explore the effect of coronavirus on cytokine production in the acute phase of the disease, plasma cytokines and chemokines (IL-2, IL-4, IL-6, IL-10, IFN-g, and TNF-a) were measured in 43 patients. (5) Radiologic assessment data (chest CT scans), such as number, size, and lesion density. (6) Treatment, including antiviral drugs, antibiotics, glucocorticoid and interferon. (7) 
Prognosis: the primary outcome was intensive care unit (ICU) admission, and the secondary outcome was the duration from hospital admission to discharge or the end of follow-up (March 16, 2020).

Two researchers independently extracted the data. If information was missing or unclear, the researchers contacted the doctor responsible for treating the patient for clarification.

\subsection{Statistical analysis}

Quantitative data are expressed as the mean (SD) and were compared using the $t$ test if normally distributed; such data are otherwise expressed as the median (inter-quartile range) and were compared using the rank sum test. Qualitative data are expressed as the frequency (\%) and were compared using the Chi-square test or Fisher exact test as appropriate. The risk factors for ICU admission were assessed using multivariate logistic regression models. With respect to duration of hospital stay, we conducted time-event data analyses where discharge was treated as the event and the follow-up duration was the time from hospital admission to discharge or the end of follow-up, whichever occurred first; the one death among our cases was treated as censored at the end of follow-up duration. We used Kaplan-Meier curves and log-rank tests for univariate analysis and used Cox proportional-hazards models to calculate the adjusted hazard ratios (HRs) with their $95 \%$ confidence intervals (Cls). We examined the proportional hazards assumption by testing the significance of interaction terms for the variable of interest and time, and found no evidence for apparent departure from the assumption of proportional hazards[10].

Statistical analyses were conducted by using SAS version 9.3 (SAS Institute, Inc., Cary, NC, USA). All tests were two-sided, and without further specification; $p<0.05$ was considered statistically significant.

\section{Results}

In this outbreak of COVID-19, 504 cases were confirmed in Wenzhou, making it the largest infected city in China outside Hubei Province. Figure 1 shows the epidemic curve of these confirmed cases (They constitute the majority of cases in Zhejiang Province). The outbreak lasted for less than one month, from the first case on January 21, to February 18 (no new case found).

Among them, 148 patients were included in the current study. In total, $19.6 \%$ of patients was severe; and other patients were moderate $(77.7 \%)$ or mild $(2.7 \%)$, these two groups were combined in the subsequent analysis.

\subsection{Demographic characteristics}

The basic demographic characteristics of the included patients are shown in Table 1. The median age was 52 years (IQR, 43-57 years; range, 10-93 years), 92 (62.20\%) subjects were male, and $60.77 \%$ were overweight or obese. The prevalence of smoking (current and ever smokers) was $14.19 \%$, and $77.70 \%$ never drank. 
Compared with non-severe patients, severe patients were older $(p=0.008)$. Severe patients also tended to have a higher rate of smoking and higher BMI, which were marginally significant in the univariate analysis.

Regarding the history of Wuhan exposure, $21.62 \%$ of patients either traveled to Wuhan or worked in Wuhan, i.e. had direct contact with Wuhan; $16.22 \%$ had family members who came back from Wuhan after the onset of the COVID-19 outbreak; $37.16 \%$ had close contact with other confirmed patients, and the remaining $25.00 \%$ did not have any known contact history. As shown in Table 1, the severe patients had a higher proportion of indirect contact with Wuhan.

\subsection{Clinical symptoms at the time of hospital admission}

There were five (3.38\%) asymptomatic patients in our study. Fever (81.76\%) and cough $(61.49 \%)$ were the most common symptoms at the time of admission, while less common symptoms included myalgia (20.27\%), fatigue (17.57\%), and pharyngalgia (17.57\%) (Table 1).

Compared with the non-severe cases, severe patients had faster respiratory rate and higher systolic blood pressure at the time of admission. The severe group had a higher proportion of fever (96.55\%) compared with the non-severe group $(78.15 \%)(p=0.021)$ but had lower rates of myalgia and pharyngalgia. The median duration from disease onset to hospital admission was 6 days (IQR: 3.5-10 days); there was no significant difference in this duration between the severe and non-severe groups.

Of the 148 patients, 62 (41.89\%) had one or more comorbidities. Hypertension (34 [22.97\%]), history of operation (18 [12.16\%]), diabetes (16 [10.81\%]), and cardiovascular disease (5 [3.38\%]) were the most common coexisting conditions. Compared with the non-severe cases, the severe patients had a higher proportion of chronic obstructive pulmonary disease (COPD) $(p=0.037)$.

\subsection{Laboratory findings at the time of hospital admission}

The mean C-reactive protein (CRP) level at the time of admission was elevated, with $67.57 \%$ of COVID-19 patients having an abnormal CRP level. Additionally, $41.89 \%$ of all patients had lymphopenia; COVID-19 patients also had elevated levels of alanine aminotransferase and aspartate aminotransferase (Table 2, sTable 1). To adjust for multiple comparisons, 0.0016 was used as the significance level in Table 2.

Compared with the non-severe cases, severe patients had significantly higher white blood cell counts and significantly higher CRP, D-dimer, ALT, AST, urea nitrogen, LDH, troponin, and myoglobin levels.

Furthermore, the lymphocyte count was significantly lower in the severe group. The oxygenation index of patients in the severe group was significantly lower than that in the non-severe group, and the abnormal rate was significantly higher than that of the non-severe group. When the data were adjusted for age, the results remained unchanged. 
Immunological data were available for 43 (21 severe, 22 non-severe) patients (Table 2). The CD $4^{+}$and $\mathrm{CD}^{+}$cell counts of the severe patients tended to be lower than those of the non-severe patients, and the interleukin levels in severe patients tended to be higher than those in the non-severe patients; however, these differences are not statistically significant.

\subsection{Radiologic assessments}

We obtained CT images for 96 of the 148 confirmed COVID-19 patients. Three confirmed patients had no sign of pneumonia on chest imaging. In patients with abnormal imaging (90 severe and 3 non-severe patients), bilateral lung involvement was observed in $76(81.72 \%)$ patients. Almost all (97.86\%) of the patients' chest CT findings showed ground glass opacity (GGO) or GGO combined with consolidation (sFigure 3). Lesion positions were near the pleura in 58 (62.37\%) patients. Air bronchogram signs and crazy paving sign were observed in $25(26.88 \%)$ and 42 (45.16\%) patients, respectively. Compared with the non-severe patients, the severe patients tended to have a wider area of lung involvement $(p<0.05$, sTable 2). Additionally, 22 (23.66\%) patients had other manifestations, such as bullae and pulmonary nodules.

\subsection{Treatments}

All patients received antiviral therapy; $65.7 \%$ used one type, and $34.3 \%$ used combined antiviral drugs. Almost all (96.8\%) patients received interferon. Additionally, 103 (69.6\%) patients received antibacterial therapy (76 used a single type of antibiotic, 27 used two types of antibiotics together). Among the 72 patients whose records included information about whether they received traditional Chinese herbal medicine, all received the Chinese medicine. Only 18.1\% (13/72) of patients used glucocorticoid therapy. For the patients who were discharged, the median treatment fee was 6615.06 RMB (IQR: 3162.358849.13 RMB).

As shown in table3, severe cases had a higher rate of using combined antiviral drugs (84.62\%) compared with the non-severe cases (29.89\%). Furthermore, the severe group also had a higher proportion of antibiotic use, higher number of antibiotics, and higher proportion of glucocorticoid use compared with the non-severe group. This may be because the severe patients usually had more symptoms at the time of hospital admission, so they were treated more aggressively.

\subsection{Prognosis}

Eight patients (5.41\%; all in the severe group) were admitted to ICU and used mechanical ventilation. One 79-year-old patient who had hypertension, diabetes, and cardiovascular disease, died of gastrointestinal hemorrhage and disseminated intravascular coagulation (DIC). As of March 16, 146 patients had been discharged, and the median duration of hospital stay was 20 days $(95 \% \mathrm{Cl}$ : $19-21$ days; IQR: $15-26$ days). sFigure 1 shows the process of discharge among these patients. 
The discharge process was slower for severe patients $(p=0.031$, sFigure 2$)$. After adjustment for age ( $>50$ years or not), gender, comorbidity, and lymphocyte count (normal or not), the results remained unchanged; the adjusted HR was 0.56 (95\%Cl: 0.35-0.90).

\subsection{Factors of prognosis among COVID-19 cases}

Based on the results of the univariate analysis, the factors considered to be professionally meaningful and considered collinearity; factors shown in Table 4 were finally included in multivariable regression models. We found that higher ALT level was independently associated with ICU admission. Additionally, older age, higher respiratory rate (beats/min), smoking, and comorbidity tended to be associated with higher risk to ICU and longer hospital stays (although these differences failed to reach statistical significance). Additionally, the combination use of arbidol and lopinavir/ritonavir did not have better prognosis than that using arbidol alone. When we restricted the analysis subset to the severe patients, we found similar results (see sTable 3).

\section{Discussion}

Although the rate of new cases of COVID-19 has gradually reduced in China, the situation abroad is becoming increasingly more serious. This report, to our knowledge, is one of the largest cohort studies of hospitalized patients with COVID-19 in China outside Hubei province. We found some specific clinical features different with previous studies of patients in Wuhan, and we also found some potential factors of ICU admission, and duration of hospital stay.

First, the symptoms of the second-generation cases were less typical compared with those in Wuhan, and $25 \%$ of patients did not have any clear contact history with Wuhan or confirmed patients. We found here that the proportion of patients with fever (81.76\%) at admission was lower than that of some earlier studies in Wuhan (98.0\%[5], 98.6\%[11]), but similar to another study in Zhejiang province (77\%)[12]. The proportion of patients with cough was also not as high as reported in other publications[6]. But consistent with other reports $[4,6,13,14]$, people of different ages (10-93 years old) could all be infected by SARSCoV-2. Moreover, we identified five asymptomatic patients (four with visible abnormalities on chest CT, one patient without any signs), which is consistent with other studies [15], but these individuals were still somewhat contagious; moreover, being asymptomatic may cause a delay in treatment, and so needs more attention. Another important finding was that $25 \%$ of our patients did not have any clear contact history with Wuhan or confirmed COVID-19 patients, which is higher than other reports $[12,16,17]$. Although some patients may have been withholding information or were unwilling to state a specific contact history, this high rate may indicate the high strength of viral infectivity. Therefore, to prevent largescale spread of the virus worldwide, it is urgent to adopt prompt and strict control measures, such as close monitoring and isolation of patients and those with close contact to patients.

Secondly, the proportion of overweight or obese among these COVID-19 patients was over $60 \%$, which is much higher than general populations[18, 19]. And the proportion of smokers (14.2\%) was also higher 
than other studies(1.4\%)[20]. Moreover, we found that the proportion of smokers in the severe group was higher than that in the non-severe group ( $26.92 \%$ vs. $11.83 \%$, respectively), which is consistent other reports[7, 20](22.1\% vs. $13.1 \%$, respectively). Although the reason for these associations needs to be explored, this finding may suggest that maintaining a healthy lifestyle and weight may lower the risk of succumbing to infectious diseases, in addition to helping prevent chronic non-communicable diseases.

Other clinical characteristics and laboratory findings in this population were consistent with previous studies. The proportion of patients with comorbidities in this study $(41.89 \%)$, was higher than that reported in a national-wide study in China (25.2\%)[7], but lower than that in other studies (46.4\%)[11]. The most common coexisting conditions were consistent with other studies[11]. This finding further confirms that people with chronic diseases, especially diabetes and hypertension, should take extra care to avoid contact with potential sources of COVID-19 infection. Laboratory findings in our study were also consistent with those of previous studies. For example, $41.49 \%$ and $2.03 \%$ of patients had lymphopenia and thrombocytopenia, respectively, which are consistent with the rates reported by a previous study in Zhejiang province $(42 \%, 3 \%)[12]$, but lower than the average national level of China $(82.1 \%, 36.2 \%)$ [7] and other studies in Wuhan[5,20]. Compared with the non-severe cases, severe cases had a higher proportion of abnormalities in C-reactive protein level, white blood cell count, lymphocyte count, and aminotransferase level, which are consistent with previous reports[7, 20].

Our study observed that most patients' chest CT findings showed GGO or consolidation; this rate is higher than those in other studies[21]. Some studies found that patients with COVID-19 infection may have abnormal imaging findings on CT before they have clinical symptoms[21, 22]. And in our study, four $(80.00 \%)$ symptomatic patients had abnormal CT imaging. The possibility that some patients with insignificant clinical manifestations could be identified through abnormal CT signs is of great significance to the early diagnosis of secondary epidemic areas. There were three patients in our study whose CT scans appeared normal but were positive for COVID-19 according to a nucleic acid test. As noted by other studies, these patients require careful attention to avoid a missed diagnosis[23].

As for treatment, the proportion of antiviral therapy reached almost $100 \%$ in this study, with $69.59 \%$ of patients receiving antibiotic treatment, similar to other studies[7]; however, the proportion of glucocorticoid therapy is significantly lower than that of previous studies (44.9\%)[11]. The median duration of hospital stay(20 days) was higher than other reports (10-13 days)[11, 24]. This difference may be due to our relatively long follow up time, during which almost all patients were discharged, whereas other studies included many patients who were still in the hospital and had high mortality.

The duration of the whole epidemic in this study was relatively short, suggesting that Wenzhou's prevention and control measures were very effective. In this outbreak, Zhejiang was the first province in China to initiate a Primary Public Health Emergencies Primary Response. As the worst-affected city in Zhejiang province, Wenzhou also adopted a series of control measures. Owing to the government's strong measures and the efforts of medical workers, all confirmed COVID-19 patients in Wenzhou as of March 16 were cleared completely. Additionally, the fatality rate of patients in Wenzhou( $0.2 \%, 1$ death among 
504 confirmed cases), was much lower than the national average (4.0\%)[25] and other findings (15\%[5], $11 \%[6])$. Furthermore, the proportion of patients transferred to the ICU $(5.4 \%)$ in our study is lower than other results $[5,7,11]$.

Interestingly, we found that increased ALT level was independently associated with the odds of admission to ICU. Moreover, older age, faster respiratory rate, smoking, decreased lymphocyte count, tended to be positively associated with ICU admission and longer hospital stay. To improve the prognosis of COVID-19 patients, doctors should pay more attention to these high-risk individuals. However, there is no evidence that the combination of arbidol and lopinavir/ritonavir had better prognosis than that using arbidol alone. As a new emerging virus, more effective treatment or vaccine are urgently needed.

Our study has several limitations. First, as an observational study, residual confounding owing to unmeasured factors might still be possible. Because repeated measures of all laboratory findings were lacking for most patients, we cannot analyze trends in their change or their effect on prognosis. Twentyfour patients were transferred to other hospitals for treatment, and subsequent treatment information was missing for these individuals. Last but not least, interpretation of our findings might be limited by the sample size. However, by including all patients in the two designated hospitals for COVID-19 in Wenzhou, we believe our study population is representative one for a city outside Wuhan.

In summary, this retrospective, multi-center cohort of patients with COVID-19 outside Wuhan provided new insight into a secondary epidemic area. The symptoms of the second-generation cases were less typical, and some patients did not have a clear contact history, which can cause a delay in treatment, as well as more infections. Lifestyle factors, such as smoking and being overweight, may play some role in the disease incidence and/or progression. Older age, smoking behavior, faster respiratory rate, increased ALT level, and decreased lymphocyte count may be positively associated with ICU admission, so individuals with these factors constitute a high-risk population. However, further studies with a longer follow-up and larger sample size need to be performed to confirm these findings. Future research on developing a vaccine and identifying effective drugs for COVID-19 are also needed.

\section{Abbreviations}

COVID-19, coronavirus disease 2019; SARS-CoV-2, severe acute respiratory syndrome-related coronavirus 2; MERS-CoV, Middle East Respiratory Syndrome Coronavirus;ICU, intensive care unit; WHO, the World Health Organization; RT-PCR, Reverse Transcription-Polymerase Chain Reaction; BMI, Body Mass Index; IL, Interleukin; IFN, Interferon; TNF, Tumor necrosis factor; CT, Computed Tomography; CRP, C-reactive protein, ALT, Alanine aminotransferase; APTT, Activated partial thromboplastin time.

\section{Declarations}

\section{Acknowledgments}


We are indebted to the coordination of Drs. Shanshan Su (The First Affiliated Hospital of Wenzhou Medical University), which greatly facilitate the collection of patient's data.

\section{Author contributions}

HY Shi, LY He, WJ Sun, JX Xu, XD Chen, WT Zhang, XW Sun, YB Hu and WP Ji collected the clinical data. HY Shi and ML Wang processed statistical data. HY Shi, LY He and JX Xu drafted the manuscript. ML Wang, HM Zeng, XY Xue and $X$ Shen revised the final manuscript. WJ Sun, $X Y$ Xue and $X$ Shen provided administrative, technical, or material support.

\section{Funding}

This study was funded by General Scientific Research Project of Education Department of Zhejiang Province (No. Y201941489), Project of the Regional Diagnosis and Treatment Center of the Health Planning Committee (No. JBZX-201903), Special fund for clinical research of Wu Jieping Medical Foundation (No. 320.6750.19010), National Natural Science Foundation of China (81972261), Zhejiang Provincial Natural Science Foundation of China (LY18H160046), Zhejiang Medical Science Foundation (2018KY532), and Science and Technology Planning Project of Wenzhou (Y20190147).

The funding bodies do not have any role in study design, data collection, data analysis, data interpretation, or writing of the report

\section{Availability of data and materials}

The datasets during and/or analysed during the current study available from the corresponding author on reasonable request

\section{Ethics approval and consent to participate}

The ethics committees of the designated hospitals approved this retrospective study. Written informed consent was waived.

\section{Consent for publication}

Not applicable.

\section{Competing interests}


None of the authors have any conflict of interest to declare.

\section{References}

[1] Zhou P, Yang XL, Wang XG, Hu B, Zhang L, Zhang W, et al. A pneumonia outbreak associated with a new coronavirus of probable bat origin. Nature. 2020;579(7798):270-3. https://doi.org/10.1038/s41586020-2012-7

[2] Del Rio C, Malani PN. 2019 Novel Coronavirus-Important Information for Clinicians [published online ahead of print, 2020 Feb 5]. JAMA. 2020;10.1001/jama.2020.1490.

https://doi.org/10.1001/jama.2020.1490

[3] World Health Organization. Coronavirus disease (COVID-2019) Situation Reports-51,

https://www.who.int/docs/default-source/coronaviruse/situation-reports/20200311-sitrep-51-covid19.pdf?sfvrsn=1ba62e57_10/; 2020 [accessed 11 March 2020]

[4] World Health Organization. Coronavirus disease (COVID-2019) situation Reports-74,

https://www.who.int/docs/default-source/coronaviruse/situation-reports/20200403-sitrep-74-covid-19mp.pdf?sfvrsn=4e043d03_14/; 2020 [accessed 3 April 2020]

[5] Huang C, Wang Y, Li X, Ren L, Zhao J, Hu Y, et al. Clinical features of patients infected with 2019 novel coronavirus in Wuhan, China. Lancet. 2020;395(10223):497-506. https://doi.org/10.1016/S01406736(20)30183-5

[6] Chen N, Zhou M, Dong X, Qu J, Gong F, Han Y, et al. Epidemiological and clinical characteristics of 99 cases of 2019 novel coronavirus pneumonia in Wuhan, China: a descriptive study. Lancet. 2020;395(10223):507-13. https://doi.org/10.1016/S0140-6736(20)30211-7

[7] Guan WJ, Ni ZY, Hu Y, Liang WH, Ou CQ, He JX, et al. Clinical Characteristics of Coronavirus Disease 2019 in China. N Engl J Med. 2020. https://doi.org/10.1056/NEJMoa2002032

[8] National Health and Health Commission of the People's Republic of China. Diagnosis and treatment guidelines for 2019 novel coronavirus pneumonia (draft version7) $\square$ http://www.nhc.gov.cn/yzygj/s7653p/202003/46c9294a7dfe4cef80dc7f5912eb1989.shtml/ ;2020 [accessed 4 March 2020]

[9] Zhou B. Predictive values of body mass index and waist circumference to risk factors of related diseases in Chinese adult population. Chin J Epidemiol. 2002;23(1):5-10.

[10] Zheng F, Liao C, Fan QH, Chen HB, Zhao XG, Xie ZG, et al. Clinical Characteristics of Children with Coronavirus Disease 2019 in Hubei, China. Curr Med Sci. 2020. https://doi.org/10.1007/s11596-0202172-6 
[11] Wang D, Hu B, Hu C, Zhu F, Liu X, Zhang J, et al. Clinical Characteristics of 138 Hospitalized Patients With 2019 Novel Coronavirus-Infected Pneumonia in Wuhan, China. JAMA. 2020.

https://doi.org/10.1001/jama.2020.1585

[12] Xu XW, Wu XX, Jiang XG, Xu KJ, Ying LJ, Ma CL, et al. Clinical findings in a group of patients infected with the 2019 novel coronavirus (SARS-Cov-2) outside of Wuhan, China: retrospective case series. BMJ. 2020;368:m606. https://doi.org/10.1136/bmj.m606

[13] Song YZ, You HY, Zhu ZH, Wen ZD, Xu HY, Chen BC, et al. The C825T Polymorphism of the G-Protein beta3 Gene as a Risk Factor for Functional Dyspepsia: A Meta-Analysis. Gastroenterol Res Pract. 2016;2016:5037254. https://doi.org/10.1155/2016/5037254

[14] Qian K, Deng Y, Tai Y, Peng J, Peng H, Jiang L. Clinical Characteristics of 2019 Novel Infected Coronavirus Pneumonia囚A Systemic Review and Meta-analysis. medRxiv. 2020:2020.02.14.20021535. https://doi.org/10.1101/2020.02.14.20021535

[15] Bai Y, Yao L, Wei T, Tian F, Jin DY, Chen L, et al. Presumed Asymptomatic Carrier Transmission of COVID-19. JAMA. 2020. https://doi.org/10.1001/jama.2020.2565

[16] Xu M, Li M, Zhan W, Han T, Liu L, Zhang G, et al. Clinical analysis of 23 cases of 2019 novel coronavirus infection in Xinyang City, Henan Province. Chinese Critical Care Medicine. 2020;32(2):E010-E.

[17] Wan S, Xiang Y, Fang W, Zheng Y, Li B, Hu Y, et al. Clinical features and treatment of COVID-19 patients in northeast Chongqing. J Med Virol. 2020. https://doi.org/10.1002/jmv.25783

[18] Zhang L, Wang Z, Wang X, Chen Z, Shao L, Tian Y, et al. Prevalence of overweight and obesity in China: Results from a cross-sectional study of 441 thousand adults, 2012-2015. Obes Res Clin Pract. 2020. https://doi.org/10.1016/j.orcp.2020.02.005

[19] Song N, Liu F, Han M, Zhao Q, Zhao Q, Zhai H, et al. Prevalence of overweight and obesity and associated risk factors among adult residents of northwest China: a cross-sectional study. BMJ Open. 2019;9(9):e028131. https://doi.org/10.1136/bmjopen-2018-028131

[20] Zhang JJ, Dong X, Cao YY, Yuan YD, Yang YB, Yan YQ, et al. Clinical characteristics of 140 patients infected with SARS-CoV-2 in Wuhan, China. Allergy. 2020:10.1111/all.14238.

https://doi.org/10.1111/all.14238

[21] Chung M, Bernheim A, Mei X, Zhang N, Huang M, Zeng X, et al. CT Imaging Features of 2019 Novel Coronavirus (2019-nCoV). Radiology. 2020;295(1):202-7. https://doi.org/10.1148/radiol.2020200230

[22] Sun Z. Diagnostic Value of Chest CT in Coronavirus Disease 2019 (COVID-19). Curr Med Imaging. 2020. https://doi.org/10.2174/1573405616999200320163751 
[23] Ling Z, Xu X, Gan Q, Zhang L, Luo L, Tang X, et al. Asymptomatic SARS-CoV-2 infected patients with persistent negative CT findings. Eur J Radiol. 2020;126:108956.

https://doi.org/10.1016/j.ejrad.2020.108956

[24] Wu C, Chen X, Cai Y, Xia Ja, Zhou X, Xu S, et al. Risk Factors Associated With Acute Respiratory Distress Syndrome and Death in Patients With Coronavirus Disease 2019 Pneumonia in Wuhan, China. JAMA Intern Med. 2020. https://doi.org/10.1001/jamainternmed.2020.0994

[25] Wu Z, McGoogan JM. Characteristics of and Important Lessons From the Coronavirus Disease 2019 (COVID-19) Outbreak in China: Summary of a Report of 72314 Cases From the Chinese Center for Disease Control and Prevention. JAMA. 2020. https://doi.org/10.1001/jama.2020.2648

\section{Tables}

Table 1 Basic characteristics at time of hospital admission for COVID-19 patients in Wenzhou, China ( $\mathrm{n}=148)$ 


\begin{tabular}{|c|c|c|c|c|}
\hline Characteristics & All $(n=148)$ & Non-severe $(n=119)$ & severe $(n=29)$ & $p$ value \\
\hline Age, years & $52.00(42.75-57.00)$ & $51.00(41.00,57.00)$ & $56.00(49.00,72.00)$ & 0.008 \\
\hline$<20$ & $1(0.68 \%)$ & $1(0.84 \%)$ & $0(0 \%)$ & \\
\hline $20-$ & $9(6.08 \%)$ & $9(7.56 \%)$ & $0(0 \%)$ & \\
\hline $30-$ & $15(10.14 \%)$ & $13(10.92 \%)$ & $2(6.9 \%)$ & \\
\hline $40-$ & $39(26.35 \%)$ & $31(26.05 \%)$ & $8(27.59 \%)$ & \\
\hline $50-$ & $52(35.14 \%)$ & $45(37.82 \%)$ & $7(24.14 \%)$ & \\
\hline 60- & $32(21.62 \%)$ & $20(16.81 \%)$ & $12(41.38 \%)$ & \\
\hline Male, $n(\%)$ & $92(62.20 \%)$ & $71(59.66 \%)$ & $21(72.41 \%)$ & 0.204 \\
\hline Body mass index, $\mathrm{kg} / \mathrm{m}^{2}$ & $24.50(3.60)$ & $24.26(3.56)$ & $26.03(3.54)$ & 0.051 \\
\hline Thin & 8 (6.15\%) & $8(7.14 \%)$ & $0(0.00 \%)$ & \\
\hline Normal & $43(33.08 \%)$ & 38 (33.93\%) & $5(27.78 \%)$ & \\
\hline Overweight & 62 (47.69\%) & $53(47.32 \%)$ & $9(50.00 \%)$ & \\
\hline Obese & 17 (13.08\%) & $13(11.61 \%)$ & $4(22.22 \%)$ & \\
\hline Smoke & & & & 0.050 \\
\hline current smoker & $17(11.49 \%)$ & $11(9.24 \%)$ & $6(20.69 \%)$ & \\
\hline past smoker & 4 (2.70\%) & $2(1.68 \%)$ & $2(6.90 \%)$ & \\
\hline Non-smoker & $127(85.81 \%)$ & $106(89.08 \%)$ & $21(72.41 \%)$ & \\
\hline Drink & & & & 0.214 \\
\hline Daily or Frequently & $14(9.46 \%)$ & $9(7.56 \%)$ & $5(17.24 \%)$ & \\
\hline Rarely or Never & $134(90.54 \%)$ & $110(92.44 \%)$ & $24(82.75 \%)$ & \\
\hline History of Wuhan exposure & & & & 0.105 \\
\hline direct contact with Wuhan & $32(21.62 \%)$ & $26(21.85 \%)$ & $6(20.69 \%)$ & \\
\hline indirect contact with Wuhan & $24(16.22 \%)$ & $15(12.61 \%)$ & $9(31.03 \%)$ & \\
\hline contact with other confirmed patients & $55(37.16 \%)$ & $46(38.66 \%)$ & $9(31.03 \%)$ & \\
\hline did not have any known contact & $37(25.00 \%)$ & $32(26.89 \%)$ & $5(17.24 \%)$ & \\
\hline Temperature, ${ }^{\circ} \mathrm{C}$ & $37.24(0.76)$ & $37.16(0.75)$ & $37.57(0.75)$ & 0.009 \\
\hline Heart rate, times $/ \mathrm{min}$ & $85.07(14.09)$ & $84.95(14.09)$ & $85.55(14.31)$ & 0.837 \\
\hline Respiratory rate, times/min & $20.18(3.31)$ & $19.58(2.62)$ & $22.62(4.58)$ & $<0.001$ \\
\hline $\mathrm{SBP}, \mathrm{mmHg}$ & $129.89(16.74)$ & $128.2(14.6)$ & $136.7(22.5)$ & 0.014 \\
\hline $\mathrm{DBP}, \mathrm{mmHg}$ & $81.32(11.69)$ & $81.60(11.83)$ & $80.17(11.22)$ & 0.558 \\
\hline \multicolumn{5}{|l|}{ Symptoms } \\
\hline Fever & $121(81.76 \%)$ & $93(78.15 \%)$ & $28(96.55 \%)$ & 0.021 \\
\hline Cough & $91(61.49 \%)$ & $70(58.82 \%)$ & $21(72.41 \%)$ & 0.177 \\
\hline Myalgia & $30(20.27 \%)$ & $28(23.53 \%)$ & $2(6.90 \%)$ & 0.046 \\
\hline Fatigue & $26(17.57 \%)$ & $21(17.65 \%)$ & $5(17.24 \%)$ & 0.959 \\
\hline Pharyngalgia & $26(17.57 \%)$ & $26(21.85 \%)$ & $0(0.00 \%)$ & 0.006 \\
\hline Nausea, diarrhea orabdominal pain & $18(12.16 \%)$ & $14(11.76 \%)$ & $4(13.79 \%)$ & 0.764 \\
\hline Running nose & $9(6.08 \%)$ & $8(6.72 \%)$ & $1(3.45 \%)$ & 0.508 \\
\hline Days from onset to admission* & $6.00(3.50-10.00)$ & $6.00(3.50-10.50)$ & $8.00(5.00-10.00)$ & 0.953 \\
\hline Comorbidities & $62(41.89 \%)$ & $49(41.18 \%)$ & $13(44.83 \%)$ & 0.721 \\
\hline Hypertension & $34(22.97 \%)$ & $26(21.85 \%)$ & $8(27.59 \%)$ & 0.510 \\
\hline History of operation & $18(12.16 \%)$ & $14(11.76 \%)$ & $4(13.79 \%)$ & 0.764 \\
\hline Diabetes & $16(10.81 \%)$ & $13(10.92 \%)$ & $3(10.34 \%)$ & 0.928 \\
\hline Cardiovascular disease & $5(3.38 \%)$ & $5(4.20 \%)$ & $0(0.00 \%)$ & 0.583 \\
\hline Chronic pulmonary disease & $2(1.35 \%)$ & $0(0.00 \%)$ & $2(6.90 \%)$ & 0.037 \\
\hline
\end{tabular}

*57 cases had missing values. Quantitative data are expressed as the mean (SD) and were compared using a $t$-test if normally distributed; otherwise, quantitative data are expressed as the median (inter-quartile range) and were compared using the rank sum test. Qualitative data are expressed as the frequency (\%) and were compared using a Chi-square test or Fisher exact test as appropriate. Age and BMI were treated as continuous when compared. 
Table 2 Laboratory findings of COVID-19 patients at time of admission in Wenzhou $(n=148)$

\begin{tabular}{|c|c|c|c|c|}
\hline Laboratory findings & All $(n=148)$ & Non-severe $(n=119)$ & Severe $(n=29)$ & $p$ value \\
\hline C-reactive protein, $\mathrm{mg} / \mathrm{L}$ & $14.66(4.58-43.08)$ & $11.42(3.74,36.55)$ & $37.30(16.70,91.99)$ & $<0.001$ \\
\hline White blood cell count, $\times 10^{9} / \mathrm{L}$ & $5.04(4.43-6.35)$ & $4.940(4.05,5.77)$ & $8.21(5.31,11.09)$ & $<0.001$ \\
\hline Lymphocyte count, $\times 10^{9} / \mathrm{L}$ & $1.21(0.89-1.53)$ & $1.29(0.98,1.56)$ & $0.71(0.50,0.99)$ & $<0.001$ \\
\hline Red blood cell count, $\times 10^{12} / \mathrm{L}$ & $4.47(0.55)$ & $4.53(0.54)$ & $4.24(0.54)$ & 0.012 \\
\hline Haemoglobin, g/dl & $134.49(17.41)$ & $135.61(17.63)$ & $129.90(15.94)$ & 0.113 \\
\hline Platelet count, $\times 10^{9} / \mathrm{L}$ & $203.50(167.25-262.50)$ & $204.0(169.0,270.0)$ & $194.0(155.0,251.0)$ & 0.152 \\
\hline oxygen saturation(day1) & $97.28(1.68)$ & $97.37(1.54)$ & $96.82(1.92)$ & 0.122 \\
\hline oxygen saturation(day2) & $97.26(2.97)$ & $97.96(1.64)$ & $95.43(4.29)$ & $<0.001$ \\
\hline APTT,$+ s$ & $40.64(36.55,45.00)$ & $41.80(38.70,45.00)$ & $36.20(34.35,41.45)$ & 0.001 \\
\hline Thrombin time,s & $15.55(14.80,16.30)$ & $15.30(14.70,15.90)$ & $16.30(15.65,17.40)$ & $<0.001$ \\
\hline Prothrombin time,s & $13.40(12.67,13.90)$ & $13.50(12.80,13.80)$ & $13.10(12.20,14.05)$ & 0.259 \\
\hline Creatinine, $\mu \mathrm{mol} / \mathrm{L}$ & $62.00(54.00,74.50)$ & $62.20(52.50,75.60)$ & $63.50(54.50,70.00)$ & 0.575 \\
\hline Blood urea nitrogen, mmol/L & $4.40(1.79)$ & $4.20(1.74)$ & $5.11(1.82)$ & 0.017 \\
\hline Lactate dehydrogenase, U/L & $378.00(308.50-460.25)$ & $374.0(287.0,410.0)$ & $459.0(332.0,520.0)$ & 0.079 \\
\hline Troponin, ng/ml & $0.012(0.011,0.013)$ & $0.012(0.012,0.012)$ & $0.014(0.007,0.030)$ & 0.088 \\
\hline Creatine kinase, $\mathrm{U} / \mathrm{L}$ & $72.00(50.50,141.00)$ & $66.00(44.00,102.00)$ & $81.0(63.0,316.0)$ & 0.026 \\
\hline Myoglobin, ng/ml & $36.00(25.00,60.70)$ & $31.90(23.00,42.70)$ & $70.0(47.8,178.1)$ & $<0.001$ \\
\hline $\mathrm{PaO}_{2} / \mathrm{FiO}_{2}$ & $391.20(321.21,479.00)$ & $452.40(355.00,490.48)$ & $332.00(248.00,378.23)$ & $<0.001$ \\
\hline Immunological indicators & All $(n=43)$ & Non-severe $(n=22)$ & severe $(n=21)$ & \\
\hline $\mathrm{CD}^{+} / \mathrm{ul}$ & $165.0(39.10,342.00)$ & $253.5(43.6,446.0)$ & $142.0(36.2,208.0)$ & 0.114 \\
\hline $\mathrm{CD}^{+} / \mathrm{ul}$ & $83.0(23.10,200.00)$ & $152.5(27.0,259.0)$ & $45.00(22.90,134.00)$ & 0.198 \\
\hline
\end{tabular}

* Quantitative data are expressed as the mean (SD) and were compared using a $t$-test if normally distributed; otherwise, quantitative data are expressed as the median (inter-quartile range) and were compared using the rank sum test.

†APTT $=$ Activated partial thromboplastin time

Table 3 Treatments of COVID-19 patients in Wenzhou, China 


\begin{tabular}{|c|c|c|c|c|}
\hline Treatment & All $(n=148)$ & Non-severe $(n=119)$ & Severe $(n=29)$ & $p$ value \\
\hline Antiviral drugs & & & & $<0.001$ \\
\hline Arbidol & $65(43.92 \%)$ & $61(51.26 \%)$ & $4(13.79 \%)$ & \\
\hline Arbidol + Lopinavir/ritonavir & $48(32.43 \%)$ & $26(21.85 \%)$ & $22(75.86 \%)$ & \\
\hline Others & $35(23.65 \%)$ & $32(26.89 \%)$ & $3(10.35 \%)$ & \\
\hline Antibiotics & & & & $<0.001$ \\
\hline Yes & $103(69.59 \%)$ & $74(62.18 \%)$ & $29(100.00 \%)$ & \\
\hline No & 45 (30.41\%) & $45(37.82 \%)$ & $0(0.00 \%)$ & \\
\hline Number of antibiotics & & & & $<0.001$ \\
\hline 0 & $45(30.41 \%)$ & $45(37.82 \%)$ & $0(0.00 \%)$ & \\
\hline 1 & $76(51.35 \%)$ & $58(48.74 \%)$ & $18(62.07 \%)$ & \\
\hline 2 & $27(18.24 \%)$ & $16(13.45 \%)$ & $11(37.93 \%)$ & \\
\hline Interferon & & & & 1.000 \\
\hline Yes & $120(81.08 \%)$ & $93(78.15 \%)$ & $27(93.10 \%)$ & \\
\hline No & $4(2.70 \%)$ & $3(2.52 \%)$ & $1(3.45 \%)$ & \\
\hline unknown & $24(16.22 \%)$ & $23(19.33 \%)$ & $1(3.45 \%)$ & \\
\hline Glucocorticoid & & & & 0.005 \\
\hline Yes & $13(13.54 \%)$ & $10(10.87 \%)$ & $3(75.00 \%)$ & \\
\hline No & 59 (61.46\%) & 59 (64.13\%) & $0(0.00 \%)$ & \\
\hline unknown & $76(25.00 \%)$ & $50(25.00 \%)$ & $26(25.00 \%)$ & \\
\hline
\end{tabular}

Table 4 Factors for prognosis of COVID-19 cases in Wenzhou, China\#

\begin{tabular}{lcccccc}
\hline \multirow{2}{*}{ Factors* } & \multicolumn{2}{c}{ Admission to ICU } & & \multicolumn{2}{c}{ Discharge and hospital stay } \\
\cline { 2 - 3 } \cline { 6 - 7 } & Crude OR $(95 \% \mathrm{CI})$ & Adjusted OR $(95 \% \mathrm{CI})$ & & Crude HR $(95 \% \mathrm{CI})$ & Adjusted HR $(95 \% \mathrm{CI})$ \\
\hline age $(\mathrm{y})$ & $1.08(1.02,1.14)$ & $1.09(0.99,1.19)$ & & $0.99(0.98,1.00)$ & $0.91(0.58,1.42)$ \\
Male & $1.02(0.23,4.42)$ & $0.83(0.09,7.86)$ & & $1.08(0.77,1.52)$ & $1.15(0.74,1.76)$ \\
\hline RR & $1.27(1.10,1.46)$ & $1.20(0.98,1.46)$ & & $0.96(0.91,1.02)$ & $0.94(0.87,1.00)$ \\
\hline Smokers & $7.24(1.65,31.65)$ & $4.61(0.48,43.84)$ & & $0.91(0.57,1.45)$ & $0.70(0.39,1.27)$ \\
\hline Comorbidity $^{2.43(0.56,10.56)}$ & $2.430 .50(0.06,4.43)$ & & $0.97(0.70,1.35)$ & $0.69(0.46,1.04)$ \\
\hline RBC $^{-}$ & $7.75(1.76,34.06)$ & $1.47(0.15,13.94)$ & & $0.79(0.49,1.27)$ & $0.69(0.37,1.31)$ \\
\hline LYM $^{-}$ & $10.82(1.30,90.36)$ & $2.50(0.19,32.30)$ & & $1.32(0.95,1.84)$ & $1.39(0.90,2.16)$ \\
\hline ALT & $18.00(3.35,96.64)$ & $10.00(1.31,76.38)$ & & $0.96(0.60,1.52)$ & $1.56(0.89,2.75)$ \\
\hline AV &.. &.. & & $0.72(0.49,1.07)$ & $1.17(0.66,2.06)$ \\
\hline
\end{tabular}

*RR: respiratory rate (beats/min), WBC: white blood cell, RBC: red blood cell, LYM: lymphocyte count, ALT: alanine aminotransferase, AV: antiviral drugs (1=Arbidol+Lopinavir/ritonavir, $0=$ Arbidol)

\# OR>1 means risk factor (because ICU admission was coded as 1 in the logistic regression), HR $<1$ means risk factor (because discharge was coded as 1 in the Cox regression). When analyzing the factors of ICU admission using a logistic regression, the AV could not be included in the model because all eight cases who were admitted to the ICU were in the second (Arbidol +Lopinavir/ritonavir) group; when analyzing the factors of duration of hospital stay, the data were stratified by case severity.

\section{Figures}




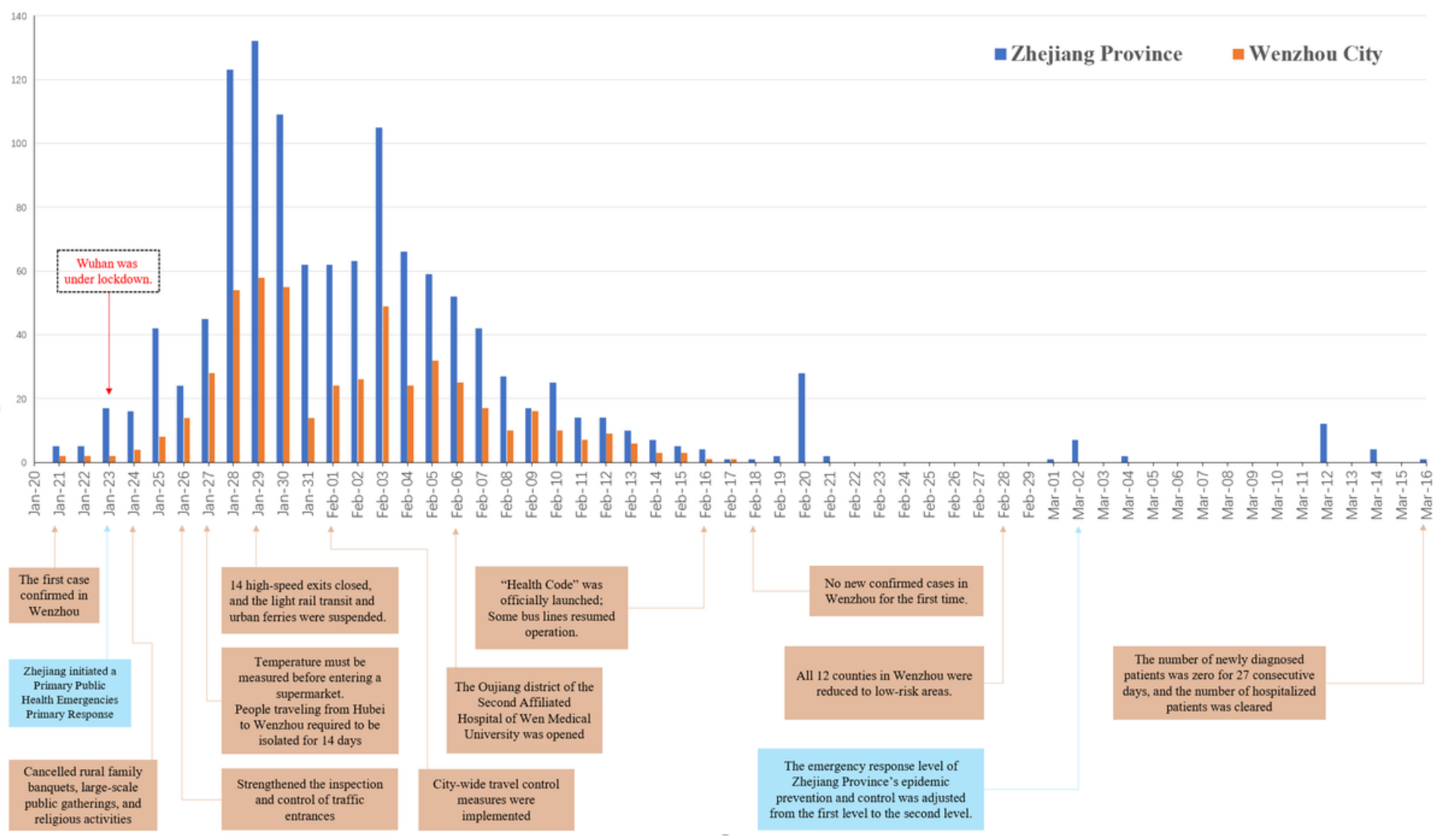

Figure 1

Epidemic curve of confirmed COVID-19 cases in Wenzhou and Zhejiang through March 16, 2020

\section{Supplementary Files}

This is a list of supplementary files associated with this preprint. Click to download.

- Supplementarytables.docx

- Supplementaryfigures2020.5.16.docx 